Document downloaded from:

http://hdl.handle.net/10251/43817

This paper must be cited as:

Lloret, J.; Canovas Solbes, A.; Rodrigues, JJPC.; Lin, K. (2013). A Network Algorithm for 3D/2D IPTV Distribution using WiMAX and WLAN Technologies. Multimedia Tools and Applications. 67(1):7-30. doi:10.1007/s11042-011-0929-4.

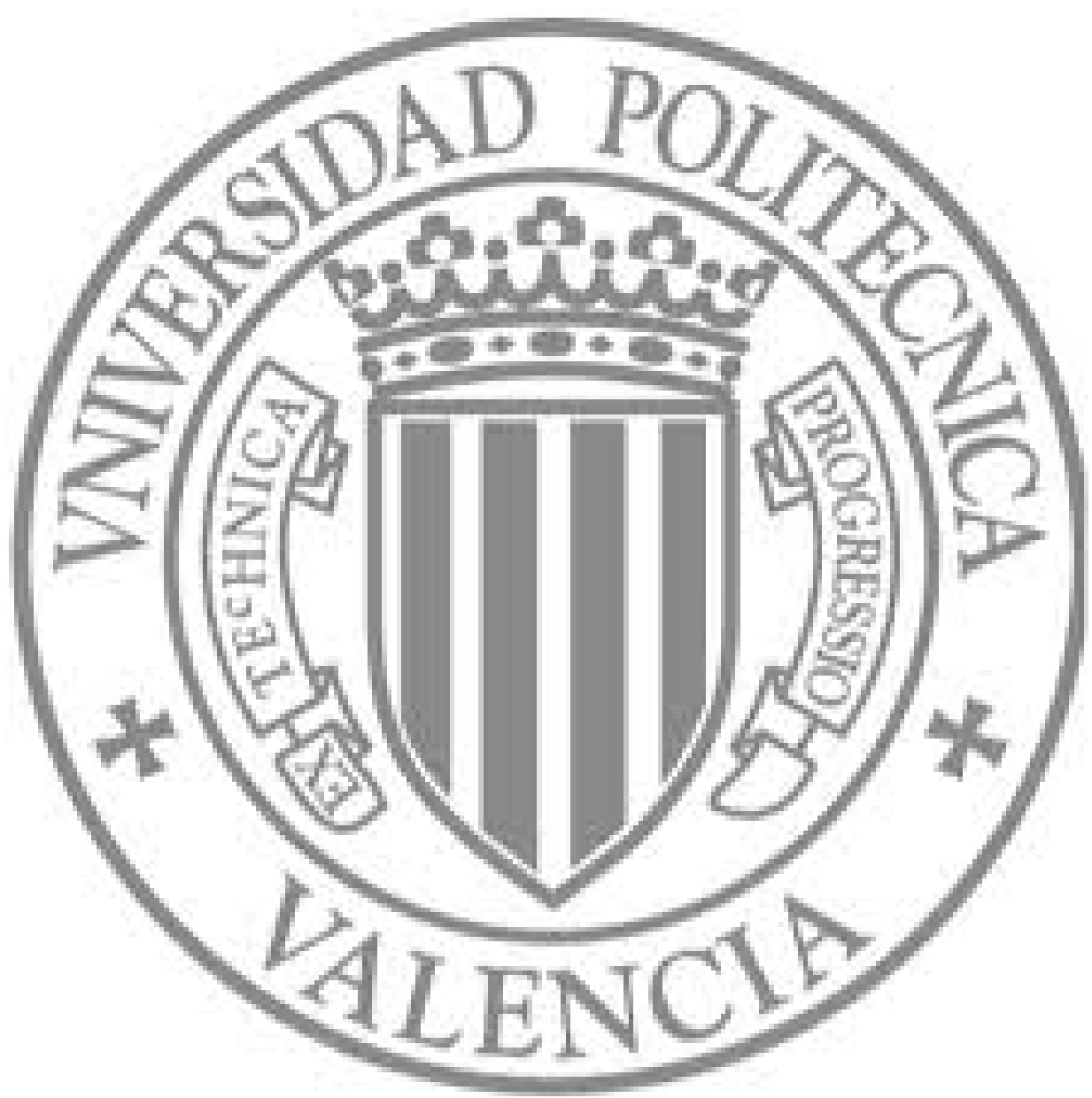

The final publication is available at

http://dx.doi.org/10.1007/s11042-011-0929-4

Copyright Springer Verlag (Germany) 


\title{
A Network Algorithm for 3D/2D IPTV Distribution using WiMAX and WLAN Technologies
}

\author{
Jaime Lloret ${ }^{1}$, Alejandro Canovas ${ }^{2}$, Joel J. P. C. Rodrigues ${ }^{3}$, Kai Lin ${ }^{4}$ \\ ${ }^{1,2}$ Integrated Management Coastal Research Institute, Universidad Politécnica de \\ Valencia, Valencia, Spain \\ ${ }^{3}$ Instituto de Telecomunicações, University of Beira Interior, Portugal \\ ${ }^{4}$ School of Computer Science and Engineering, Dalian University of Technology, \\ China \\ 1jilloret@dcom.upv.es, ${ }^{2}$ alcasol@posgrado.upv.es, ${ }^{3}$ joeljr@ieee.org, ${ }^{4}$ link@dlut.edu.cn
}

\begin{abstract}
The appearance of new broadband wireless technologies jointly with the ability to offer enough quality of service to provide IPTV over them, have made possible the mobility and ubiquity of any type of device to access the IPTV network. The minimum bandwidth required in the access network to provide appropriate quality 3D/2D IPTV services jointly with the need to guarantee the Quality of Experience (QoE) to the end user, makes the need of algorithms that should be able to combine different wireless standards and technologies. In this paper, we propose a network algorithm that manages the IPTV access network and decides which type of wireless technology the customers should connect with when using multiband devices, depending on the requirements of the IPTV client device, the available networks, and some network parameters (such as the number of loss packets and packet delay), to provide the maximum QoE to the customer. The measurements taken in a real environment from several wireless networks allow us to know the performance of the proposed system when it selects each one of them. The measurements taken from a test bench demonstrate the success of our system.
\end{abstract}

\section{Keywords}

IPTV, WiMAX, WLAN, Wireless Access Network.

\section{Introduction}

Triple play [1] and Quad play [2] are integrated services performed over IP protocol. On one hand, triple play integrates three services: voice, high-speed data and television. On the other hand, quad play is the Triple play plus the user mobility. In order to support these services properly, the networks are evolving according to Next-Generation Networks (NGN) architectures [3]. NGN describe the key architectural evolutions in telecommunication core and access networks. The general idea of NGN concept is that the network transports all information and services, such as voice, data, and video, by encapsulating them into packets. NGNs are commonly built around the 
Internet Protocol. In addition, the transport must be completely independent of the used network infrastructure. NGN take into account the quality of service (QoS) [4] to provide multimedia services with an acceptable quality over non-connection oriented networks and which do not provide quality of service.

We must not forget that new technologies and services are fostering the development of business models for TV delivered over IP [5]. According to the ITU [6], this service must possess an adequate level quality of service, security, interactivity and reliability. Therefore, IPTV service must have a correct Quality of Service (QoS) and adequate Quality of Experience (QoE) to meet end users needs.

We define IPTV network as the joint of several broadband networks that are capable to support the required bandwidth for video delivery. In addition, IPTV network topology can be split into 5 main parts: network header, core network, distribution network, access network and customer network. Generally, in an IPTV network, the video and audio streams are sent in MPEG [7] packages through RTP (Real-time Transport Protocol). Often, this protocol is used in streaming systems, along with RTSP (Real-time Transport Streaming Protocol). RTP protocol supports realtime media streaming, with control mechanisms, in order to synchronize different audio and video flows. RTP sequences the data, making possible to detect missing packets, but it does not provide guarantee in the video delivery.

The network header is responsible of delivering video and content thought the service provider network. It is essentially the core components of the infrastructure layer and the main point of the infrastructure. The devices, which are part of this network, receive, transform and distribute the content to the subscribers. It receives the subscriber requests and provides content to the set-top boxes. The network header is the most critical point of the IPTV network. For this reason, several actions should be taken into account to ensure that it has a controlled access, because only authorized users should exchange information with it.

The backbone network distributes the video flows from the header to the distribution network. It interconnects service providers and the IPTV applications with the service providers. The technologies that are often used in the backbone network are: Gigabit Ethernet, SONET/SDH, and xWDM technologies. The architectures and topologies that may have this part of the network are: point to point, ring, double ring, etc. and must be scalable. In the IPTV backbone network, the routing and switching between the aggregation routers and end routers are the most important devices of the network infrastructure. The network must have high-performance devices and should be able to mix interfaces.

The distribution network joins the end of the backbone network with the aggregation router (beginning of the access network). Its main function is to multiplex the of different service providers and to adapt the transport system to the specific characteristics of the subscriber loop. Therefore, the distribution network must perform data transmission and switching tasks efficiently. The elements that transport the multimedia content to the end user form the access network. This network manages the user demands by using the return channel. The main requirement of an access network is to have enough bandwidth to support multiple IPTV channels for each subscriber, while allows other services such as IP telephony and data. Currently the most used 
access technologies are xDSL and FTTx. These types of technologies allow large bandwidth, but these technologies do not contribute to the end user with the mobility characteristic, an important aspect in the next quad-play services. Summarising, IPTV channels transmission is sent from the server, using multicast groups, to the distribution network and, then, to the end user through the access network.

Finally, the customer network enables communication and information exchange between the computers placed in that network and the service provider network. It allows accessing the available resources in the IPTV network. The shared medium in the customer network may be wired or wireless technologies such as FastEthernet and Wi-Fi (IEEE 802.11a/b/g/n). The residential gateway connects the customer network with the service provider network.

As we have aforementioned, xDSL and FTTx do not contribute to the end user with mobility feature. For this reason, in this paper we propose a network algorithm that allows multiband devices to select the best wireless network in order to receive the best 3D/2D IPTV QoE at the end user. The system proposed uses a formula which has been deduced using the measurements taken from a real environment. A comparison between the QoE parameters will show which wireless network is preferred for IPTV devices when the place is covered by several of these technologies. Results show that our proposal is a feasible solution that could be used by the IPTV providers to provide quad-play services.

The remainder of this paper is organized as follows. Section 2 presents some related works about the IPTV transmission via wireless technologies. Section 3 explains the wireless technologies used in our system. Our algorithm proposal, the proposed protocol and architecture are described in section 4. Section 5 shows real measurements to see the wireless networks' performance and QoE. Section 6 concludes the paper and gives our future works.

\section{RELATED WORKS}

Usually, IPTV service providers use wired technology in their IPTV access network. But, there are some works where the authors propose to transmit IPTV on high-capacity wireless networks, as for example WIMAX, such as it is explained in paper [8]. In this paper, an implementation to transmit IPTV over WiMAX is presented. The authors identify some challenges and they present possible solutions about this subject. Another work where the authors examine the possibility of IPTV access network using WiMAX is [9]. In this paper, in addition to analyze the key factors and challenges presented by the technology, the authors analyzed the IPTV distribution through WiMAX in environments where the user has mobility.

Paper [10] presents an extended overview of WiMAX and the applications it can support (such as IPTV service). The authors look at the technology behind WiMAX and networks design and deployment factors that impact WiMAX coverage. The paper also compares WiMAX with two enhanced third generation (3G) technologies that are potential competitors to WiMAX. The authors claim that IPTV enables a WIMAX service provider to offer the same programming as cable or satellite TV service providers. They also describe the business models in WiMAX and state some of the benefits and drawbacks of a mobile WiMAX network. They concluded that WIMAX is an excellent complement to other wireless technologies that is WIFI. 
In [11], the authors propose a utility-based resource allocation scheme for layer-encoded IPTV multicast streaming service over IEEE 802.16 WiMAX networks. Unlike existing utility-based schemes, this mechanism is designed for wireless networks which support adaptive modulation and coding. Each video stream (or program) is encoded into different layers. Then, their mechanism adjusts the number of each user's received layers dynamically according to its channel condition and the available network bandwidth, so as to maximize total utility.

In paper [12], the authors present the IEEE 802.11 technology as the adequate to carry out the IPTV transmission. This work shows the features that should meet the IEEE 802.11 networks. On the other hand, the authors give some ideas to improve the QoS level.

In [13], the authors present an interesting architecture design for distributing triple play services over a wireless mesh in-home network. In [14], the same authors propose a wireless and wired network architecture based on in-home IPTV distribution. They develop an analytical framework for quantifying the admission region of home networks, which reveals the relationship among system and QoS parameters. The obtained results can be very important because they can help to plan future home networks.

Another work related with IPTV distribution over wireless mesh architectures is shown [15]. In this paper, authors give an overview of the possible wireless mesh architectures that could be applied in IPTV environments. They analyzed and evaluated the possibility of distributing triple play services in an indoor environment using IEEE 802.11b/g mesh network. Moreover they developed a model that was simulated in order to study which architectures were suitable to fit the appropriate QoS levels.

There are many works in the related literature in which the authors present architectures or new connection systems based mainly on QoS levels. On one hand, paper [16] presents a wireless broadband architecture that supports QoS in IPTV. This architecture is adapted to the network state by using a QoS control mechanism. On the other hand, in order to provide a QoS-guaranteed IPTV service, the authors of [17] proposed a network mechanism where the connection admission control is controlled according to the remained bandwidth. If the bandwidth is enough to allocate a new flow, a connection will be provided. Once the connection is established, it might be certainly guaranteed. The problem is that these policies cannot be applied when there are several traffic classes which have different levels of QoS. Moreover, in [18], the authors propose a QoSguaranteed IPTV service similar to the previous work. They propose this service provisioning by using a differentiated traffic handling in home network IEEE 802.11e/g Wireless LAN. In order to provide guaranteed QoS in the inter-mixed and congested traffic the authors propose a traffic engineering scheme that prioritizes the IPTV traffic. This prioritization of traffic is provided by assigning differentiated access category to each packet according to a predefined QoS class.

In [19], the authors study the IPTV mobility in WLANs. They explain that in a congested WLAN situation a substantial packet delay and packet loss can performed. They showed that jitter can be used to determine the level of congestion in a WLAN, and that it can also be used to determine which stream should be dropped during a soft handover. Moreover, they show how a stationary client can apply it in a congested WLAN in order to determine when to handover. They also describe the scheme that should be implemented at the client side. 
The authors of [20] explain an implementation experience about IPTV home networking using wireless mesh collaborative networks. In this case they packetize a H.264 video stream into several frame types of different importance levels. The H.264 packets are mapped to higher or lower priority based on their smart Enhanced Distributed Channel Access (EDCA) implementation. This approach makes the delay and packet loss probability of important packets remain low and this significantly improves the end-to-end video quality over multiple hops. Their experiments show that their smart prioritization scheme helps to improve the contention in the network and preserves the bandwidth provided to the background data traffic.

The way to improve the QoS of the transmitted video over IEEE 802.11 WLANs is studied in paper [21]. The authors used Evalvid with NS-2 simulator in order to evaluate the QoS of the delivered video over the IEEE 802.11n with frame aggregation. In order to achieve this aim, first they transmitted multiple video streams through an access point in order to check if it is possible to improve the quality of H.264 video sequences. Then, they check if this multiple video streams are affected when they add a package group for one or more users. The results show that there is a significant improvement in the video quality (VQ) and the introduction of frame aggregation improves the packet loss rate and the packet delay. Another study where IPTV is delivered through IEEE 802.11n wireless network is shown in [22]. The new features of MAC protocol proposed in IEEE 802.11n are analysed in this work. Among various successful enhancement mechanisms, the authors focus primarily on frame aggregation and bidirectional transmission. This study serves to test and tries to improve the access to voice and video services. Moreover, in [23] some of the authors of this paper studied the performance in terms of delay, jitter and packet loss when IPTV is being delivered through an IEEE 802.11n wireless local area network.

Finally, there are several research papers where the authors propose the use of several wireless technologies (e.g. WiMAX and IEEE 802.11a/b/g) for accessing the IPTV network. Moreover, the appearance of WiFi/WiMAX integrated antennas [24] make possible the creation of multiband algorithms to roam between different wireless technologies.

The authors of [25] present the important features of WIMAX technology and elaborated a comparison of WIMAX with other wireless technologies such as WI-Fl and 3G. They state that WI-MAX is delivering broadband wireless access to the masses and represents alternative to digital subscriber lines (DSL) and cable broadband access. It will provide anywhere, anytime connectivity. They proposed the coexistence of them to provide for multimedia content. Moreover, paper [26], state that hybrid networks based on systems such as WiMAX and WiFi can combine their respective advantages on coverage and data rates, offering a high Quality of Service (QoS) to mobile users. Authors state that WiFi/WiMAX dual mode terminals should seamlessly switch from one network to another, in order to obtain improved performance or at least to maintain a continuous wireless connection. They propose a new user centric algorithm for performing handover between the wireless technologies, which combines a trigger to continuously maintain the connection and another one to maximize the user throughput. They demonstrated through simulations that the algorithm implemented in existing standard technologies like 802.11 and 802.16 raises the system capacity, thus increasing the gain that can be achieved with a WiMAX and WiFi heterogeneous deployment. Moreover, some authors of this paper proposed in [27] a 
system that decides which type of wireless access network to connect with (for dual-band and triband devices) depending on the requirements of the IPTV client, the available networks, and some network parameters (such as the number of loss packets and packet delay).

Paper [28] shows the design and implementation of WiMAX and WiFi wireless networks to provide internet access to the citizens of the Loja and Zamora Chinchipe provinces (Ecuador). They only provide a plan to maximize the coverage area, but they do not propose any system to improve the performance in dual mode terminals.

Some of the works present systems that are based on delivering the IPTV signal with a particular QoS, but, as we have seen previously, this starting point is not enough. On the other hand, the papers, which propose several coexisting wireless technologies to provide network access, do not provide a network algorithm that lets the customer roam between them or are not focused on IPTV delivery, so there is no system in existence working with this feature. For this reason, we propose a network algorithm for 3D/2D IPTV distribution in the access network (using wireless technologies) based on the QoE levels defined by the ITU. This paper is an extension and enhancement of the paper presented in a conference [27]. Now we have added IEEE 802.11n to the proposed algorithm, we have improved the algorithm in order to enhance the decision to select the appropriate wireless network and we have added roaming tests between wireless technologies.

\section{Wireless Access Technologies Included in the Algorithm.}

Wired network represents high installation costs in certain areas where these high costs do not provide enough benefits or are not justified. Sometimes it is very difficult to carry xDSL technologies to these areas. On the other hand, Mobile technologies only allow the data transfer with acceptable quality, but they have some problems to guarantee real time transfer of multimedia content. For these reasons, in this point we introduce the wireless technologies that are included in our algorithm proposal. They are selected according to broadband feature because of the 3D/2D IPTV bandwidth requirements. We will overview the main characteristics of each technology (WiMAX, IEEE 802.11a, IEEE 802.11g and IEEE 802.11n), and, finally, we will compare them.

\subsection{WiMAX}

WiMAX (Worldwide Interoperability for Microwave Access) is a broadband wireless standard (published as IEEE 802.16) created for the wireless local loop and the metropolitan area [29]. It allows the data reception and broadcast by radio waves providing a shared access with several repeaters. This standard can offer coverage areas up to $50 \mathrm{~km}$ radius and speeds up to $70 \mathrm{Mbps}$ (both theoretical values). WiMAX technology is very robust and flexible, so it can work in several environments. It can withstand the multipath effects caused by the wave reflections. WiMAX can work with different channel sizes and different methods to offer two-way communications. The first version of the IEEE 802.16 standard specified a physical layer operating in the 10 to $66 \mathrm{GHz}$ range. 802.16a and 802.16-2004 added specifications for the 2 to $11 \mathrm{GHz}$ range.

A WiMAX system is composed of two main components: The WiMAX tower (base station) and the WiMAX receiver (network interface card). There are two main variants in the IEEE 802.16 
standard: the fixed access variant (IEEE 802.16d), which offers a radio link between the base station and the customer device (real implementations show $20 \mathrm{Mbps}$ for up to $6 \mathrm{Km}$ ) and the mobile variant (IEEE 802.16e), which offers a GSM/UMTS like access. The WiMAX network may have several base stations and associated antennas that communicate wirelessly with a large number of customer devices (point to multipoint connection). Each base station offers a wireless coverage on an area called cell. Although the maximum radius of each cell is theoretically about 50 kilometres, normally the typical deployments use radius cells between 3 and 10 kilometres. In our case we will include in our proposal the IEEE 802.16a standard.

Table 1 shows a summary of the main features of the most used WiMAX standards.

Table 1. Main characteristics of WiMAX standards.

\begin{tabular}{|c|c|c|c|}
\hline & 802.16 & 802.16d & 802.16e \\
\hline $\begin{array}{l}\text { Frequency } \\
\text { band }\end{array}$ & 10 to $66 \mathrm{GHz}$ & 2 to $11 \mathrm{GHz}$ & $<6 \mathrm{GHz}$ \\
\hline Operation & LOS & NLOS & NLOS \\
\hline Bit rate & $\begin{array}{l}\text { 32-134 Mbps with } \\
\text { channels of } 28 \mathrm{MHz}\end{array}$ & $\begin{array}{l}\text { Up to } 75 \text { Mbps with channels of } 20 \\
\qquad \mathrm{MHz}\end{array}$ & $\begin{array}{l}\text { Up to } 15 \text { Mbps with channels of } 5 \\
\qquad \mathrm{MHz}\end{array}$ \\
\hline Modulation & QPSK, 16QAM y 64 QAM & $\begin{array}{c}\text { OFDM with } 256 \text { subcarriers QPSK, } \\
\text { 16QAM, 64QAM }\end{array}$ & Equal than 802.16a \\
\hline Mobility & Fixed system & Fixed system & Mobile system \\
\hline Bandwidth & 20,25 y $28 \mathrm{MHz}$ & Select between 1,25 y $20 \mathrm{MHz}$ & $\begin{array}{l}\text { Equal than } 802.16 a \text { with uplink } \\
\text { channels to save power }\end{array}$ \\
\hline $\begin{array}{l}\text { Typical cell } \\
\text { radius }\end{array}$ & 2 - 5 km aprox. & $\begin{array}{l}5 \text { - } 10 \text { km aprox. } \\
\text { (50 km maximum) }\end{array}$ & 7 - 8 km aprox. \\
\hline
\end{tabular}

\subsection{IEEE 802.11a}

IEEE 802.11a was approved in 1999 [30]. It uses the OFDM (Orthogonal Frequency Division Multiplexing) modulation with 52 subcarriers in a $16.25 \mathrm{MHz}$ band. 48 of them are used for the data transmission and 4 are pilot tasks. The frequency width of each subcarrier is $312.5 \mathrm{KHz}$. Each subcarrier may be modulated by BPSK (Binary Phase Shift Keying), QPSK (Quaternary Phase Shift Keying), 16-QAM (Quadrature Amplitude Modulation) or 64-QAM. This standard gets a theoretical speed up to $54 \mathrm{Mbps}$. The transmission rate decreases when the signal quality is low. The 54 Mbps can be decreased to 48, 36, 24, 12, 9 and 6 Mbps.

IEEE 802.11a provides 12 non-overlapping channels. As it uses the $5 \mathrm{GHz}$ band, the signal has less interference than the IEEE 802.11b standard. But the equipment must be in the line of sight (LOS) of the client in order to gain a better efficiency in communications. In these frequencies the signal absorption coefficient affects more. Its architecture is based on two main components: The access points (APs), which are the base stations for the wireless network, and the wireless clients, which can be mobile devices such as laptops, personal digital assistants, IP phones, or fixed devices such as desktops and workstations that are equipped with a wireless network interface. 


\subsection{IEEE $802.11 \mathrm{~g}$}

IEEE 802.11g standard appeared in 2003 [31]. It is an evolution of the IEEE 802.11b standard. It works on $2.4 \mathrm{GHz}$ frequency band and it is compatible with IEEE 802.11b. Its theoretical transfer is $54 \mathrm{Mbps}$, although it is reduced when the receiver moves away from the AP in a real scenario. It is also decreased when the signal quality decreases. Data transmission rates are $54,48,36,24,18$, 12, 9 and 6 Mbps. The modulation scheme used in 802.11g for this data rates is OFDM, such as in 802.11a, and reverts to CCK (like the 802.11b standard) for 5.5 and 11 Mbps and DBPSK/DQPSK+DSSS for 1 and 2 Mbps. Because IEEE 802.11g uses the same radio signalling (CCK) as IEEE 802.11b at the lower four IEEE 802.11g data rates, it is fully backward compatible with IEEE 802.11b. This enables networks IEEE 802.11g to continue supporting IEEE 802.11b enabled devices when migrating to the higher performance standard. IEEE 802.11g seems to be the competence of IEEE 802.11a, but most products include both technologies because they are complementary.

IEEE 802.11g suffers from the same interference problems such as IEEE 802.11b, because both work in the already crowded $2.4 \mathrm{GHz}$ range. Additionally the success of the standard has caused density problems related to crowding in urban areas.

Although IEEE 802.11b has been more widely used than IEEE 802.11a, several variants have been appeared to improve their characteristics. The fact of operating in different bands allows them to be used at the same time. This allows 802.11g to complement IEEE 802.11a by adding three additional channels in the $2.4 \mathrm{GHz}$ band to the existing IEEE 802.11a channels. This creates more network capacity to allow for additional users. Both technologies have advantages that, when they are used in combination, offer an even stronger product. Another advantage of 802.11a is that the 5 $\mathrm{GHz}$ band has more capacity around the world. One of variants was IEEE 802.11 Super G [32]. It is Atheros' proprietary frame-bursting, compression and channel bonding technology to improve IEEE 802.11g wireless LAN performance. It duplicates the speed and throughput of the IEEE 802.11g standard, thus is able to provide $108 \mathrm{Mbps}$. Typical maximum end-user throughput ranges from approximately $40 \mathrm{Mbps}$ to $60 \mathrm{Mbps}$. Super $\mathrm{G}$ is very helpful to the users that require additional bandwidth (which is required for IPTV customers).

We should bear in mind that IEEE 802.1a/b/g variants do not provide enough bandwidth for several IPTV channels, especially when HDTV is being transmitted, but IEEE 802.11n variant is able to provide higher bandwidth.

\subsection{IEEE 802.11n}

IEEE 802.11n is the IEEE 802.11 variant that offers the highest data throughput and link range. It was ratified in September 2009. Its stronghold is based on use of the Multiple-Input MultipleOutput (MIMO) technology, which uses multiple antennas at both the transmitter and receiver to improve communication performance. This allows transmitting multiple independent data streams simultaneously in order to increase the spectral efficiency. Moreover, in IEEE 802.11n, the channel size is increased from 20MHz (given in previous IEEE 802.11 variants) to $40 \mathrm{MHz}$. As a result of these improvements, plus a frame aggregation to the Medium Access Control (MAC) 
layer, IEEE 802.11n can transmit up to $600 \mathrm{Mbps}$ with a coverage range up to 70 meters for indoor and up to 250 meters for outdoor. It uses OFDM and it is able to work with the following data rates: $7.2,14.4,15,21.7,28.9,30,43.3,45,57.8,60,65,72.2,90,120,135$, and 150. IEEE 802.11n can work at 2.4 or $5 \mathrm{GHz}$, but in order to achieve the maximum performance, $5 \mathrm{GHz}$ network is recommended. The $5 \mathrm{GHz}$ band has substantial capacity due to many non-overlapping radio channels and less radio interference as compared to the $2.4 \mathrm{GHz}$ band.

\subsection{Technology comparison}

Those four wireless technologies have many differences, but all these networks allow connections with higher bandwidth than 24 Mbps. They are shown in Table 2. In this table we analyze their frequency band, average speed, modulation, channel bandwidth, coverage radius, unlicensed spectrum, radio interference, introduction cost, device cost, mobility, current use, QoS level and security.

Table 2. Wireless Technology comparison.

WiMAX (IEEE 802.16a) WLAN (IEEE 802.11a) WLAN (IEEE 802.11g) WLAN (IEEE 802.11n)

\begin{tabular}{|c|c|c|c|c|}
\hline Frequency band & $<11 \mathrm{GHz}$ & $5 \mathrm{GHz}$ & $2,4 \mathrm{GHz}$ & 2.4 or $5 \mathrm{GHz}$ \\
\hline Average speed & Up to $70 \mathrm{Mbps}$ & $54 \mathrm{Mbps}$ & $54 \mathrm{Mbps}$ & Up to $600 \mathrm{Mbps}$ \\
\hline Modulation & $\begin{array}{c}\text { OFDM, QPSK, 16QAM } \\
\text { and 64QAM }\end{array}$ & OFDM & DSSS, CCK, OFDM & $\begin{array}{c}\text { OFDM, BPSK, QPSK, } \\
\text { 16QAM and 64QAM }\end{array}$ \\
\hline Channel bandwidth & $20 \mathrm{MHz}$ & $20 \mathrm{MHz}$ & $20 \mathrm{MHz}$ & $40 \mathrm{MHz}$ \\
\hline Coverage radius & 5-10 Km outdoor & $\begin{array}{c}35 \mathrm{~m} \text { indoor and } 95 \mathrm{~m} \\
\text { outdoor }\end{array}$ & $\begin{array}{c}38 \mathrm{~m} \text { indoor and } 100 \mathrm{~m} \\
\text { outdoor }\end{array}$ & $\begin{array}{c}70 \text { m indoor and } 250 \\
\text { meters outdoor }\end{array}$ \\
\hline Unlicensed spectrum & No & $\begin{array}{c}\text { Yes (it depends on } \\
\text { countries) }\end{array}$ & $\begin{array}{c}\text { Yes (it depends on } \\
\text { countries) }\end{array}$ & $\begin{array}{l}\text { Yes (it depends on } \\
\text { countries) }\end{array}$ \\
\hline Radio Interference & Low & Low & High & Low \\
\hline Introduction cost & High & Medium-Low & Low & Low \\
\hline Device cost & High & Medium-Low & Low & Medium-Low \\
\hline Mobility & No & Yes & Yes & Yes \\
\hline Current use & Low & Medium & High & Medium \\
\hline QoS level & High & Medium & Medium & Medium \\
\hline Security & High & Medium & Medium & Medium \\
\hline
\end{tabular}

\section{Architecture and Network Algorithm}

Service providers must be aware of the bandwidth limitations and the bottleneck in their networks. An IPTV network design and deployment must take into account the functionality, interoperability, performance, and scalability (customer growing factor). A regular IPTV can be formed by 1 or 2 super-headers, between 10 and 100 offices with video readers and more than a million of customers. It is important to provide high bandwidth for multiple video channels for standard definition (SD) and high definition (HD), data services and voice (triple-play services). Moreover, mobility should be provided (quad play services).

The service provider is responsible for the IP QoS from the network header to the residential gateway; however, the transport is performed to the set-top box of the customer. QoS has a hish impact on the operational costs. A poor QoS could imply the increase of complaints and calls from 
the customers. To solve each call imply an economical cost to the provider. Many calls mean that many customers are disgruntled (it could be worst if it happens during a sport event).

IPTV QoE parameter is defined as how good the video service satisfy the expectative of the users. It has to be equal or better than the one offered by the satellite or cable TV service providers. But it is influenced by several commercial factors such as the price, content or service characteristics, and by technical factors such as the channel change response time, quality of video, etc. Service providers must guarantee QoE in their networks by developing delay sensitive IPTV and VoD applications. The customers of IP video services do not tolerate the delay and the degradation of the quality of the video, so the customer must have high QoE. In order to guarantee IPTV QoE, the service provider should use the appropriate test tools. They must be flexible, scalable and have to give a good view of the quality in the small and the big scale. The service providers should measure the performance statistics of the lower ISO layers of the network, and don't neglect the network header and the customer network. The knowledge of how performs all the IPTV network is not enough to guarantee QoE. The standards recommend the administrators to analyze also the frame headers and the payload. The service provider must check the quality of the video and audio streams sent through an active and passive analysis to guarantee satisfactory QoE levels. The tests should be performed including all triple-play services in order to know how other traffic interfere in the quality of service of the tested one. The most common QoE parameters are shown in table 3.

Table 3. QoE Parameters.

\begin{tabular}{ll}
\multicolumn{1}{c}{ Parameter } & \multicolumn{1}{c}{ Description } \\
\hline Bandwidth & It is the minimum bandwidth guaranteed by the operator to the customer. \\
Availability & Round trip average delay. \\
Round Trip Delay & Time needed to leave from a channel and receive the new channel. \\
Zapping time & Maximum number of packets lost (but the user do not have to exceed the committed rate). \\
Packet loss & Fluctuation that occur in the round trip average delay. \\
Jitter & $\begin{array}{l}\text { Time needed in the transport layer to deliver the video stream to the final set-top box. } \\
\text { It is the quality of the video. It depends on the error correction of the codec used and the } \\
\text { belay }\end{array}$ \\
Video Quality & $\begin{array}{l}\text { It is the quality of the audio. It depends on the error correction of the codec used and the } \\
\text { bitrate used for compression. Packet losses, Jitter and latency affect to the audio quality. }\end{array}$ \\
Audio Quality &
\end{tabular}

From the customer perspective, the QoE is based on the subjective perception of the received service. Based on it, QoE could include more parameters such as (1) content availability, (2) election, access easiness and available content indexation, (3) video and audio resolution, (4) subtitles synchronization and clean audio, (5) user interface, (6) colors palette, ergonomics, navigation, design, (7) electronic program guide and (8) program description, genre classification, updates.

In order to provide a QoE measurable by the information taken from the network, we have decided to include the bandwidth, Jitter, Delay and Packet loss in our algorithm. We will see in the test bench that they provide enough information to offer the appropriate decisions, but more parameters can be added in order to fine the algorithm. 


\subsection{Architecture Description}

We propose a system where several wireless technologies coexist and the customers have multiband devices. That is, the customers' devices can join several wireless networks such as the ones explained in section 3. We think that it is a feasible idea since the authors of reference [26] demonstrated in that work that the devices can roam very fast from one wireless technology to another without having high impact to the QoS. Figure 1 shows an example of a wireless access network. In this case, customer devices are able to connect to IEEE 802.16d, IEEE 802.11a, IEEE 802.11g IEEE 802.11n at any time depending on its placement, but it could be a situation where there are places covered only by two or one of those technologies. All these wireless networks are connected to a common IPTV network infrastructure and they are able to offer 3D/2D IPTV service. The customer's device is able to measure the RSSI (Radio Signal Strength Indicator) of each available wireless network, which is stored in a list, and select the one highest RSSI.

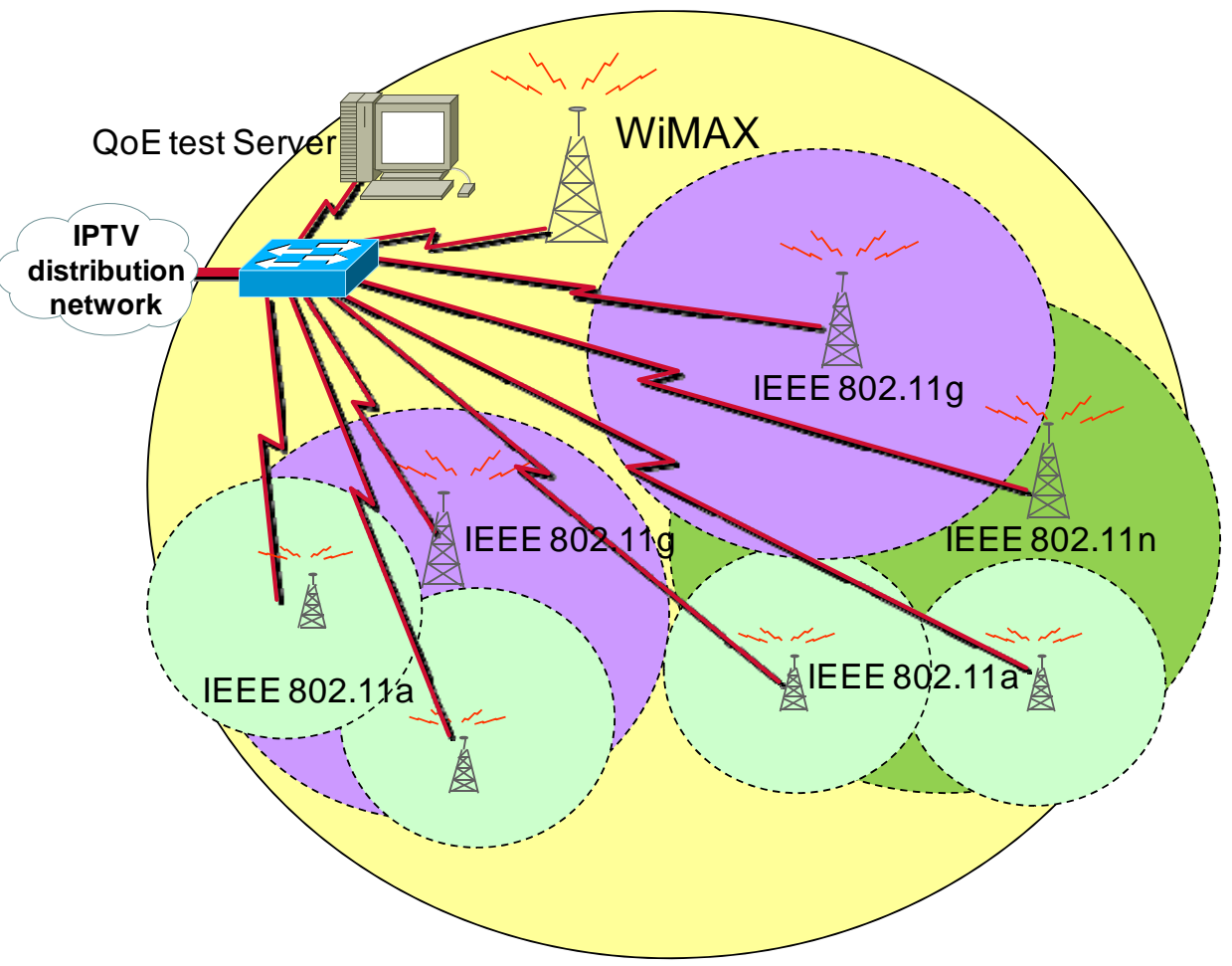

Figure 1. Multiband architecture for IPTV network access.

\subsection{Network Algorithm}

The first issue we must solve is how to differentiate the QoE for each network based on some measurable parameters. In order to define the network's QoE parameter, we looked at the Delay and Jitter values and we saw that they have similar values. When they are high, the network's QoE parameter should have a low value. On the other hand, although packet losses are very bad for the QoE, they could be zero, so it cannot directly multiply to the dividend. Moreover, higher values of packet losses affect more to the QoE value, so the $e$ number gives us the appropriate expression. 
Taking into account the aforementioned considerations, network's QoE parameter is defined as it is shown in expression 1.

$$
Q o E=\frac{1}{\left(\text { Delay }+K_{1} \cdot \text { Jitter }\right) \cdot e^{\text {PacketLoss }}}
$$

Where $K_{1}$ let us give higher importance to the Jitter parameter vs. the delay in the users' QoE calculus. None of the parameters used in the expression could be negative. In figure 2 we show QoE values as a function of the delay of the network, for several jitter. We have fixed $K_{1}=2$ and PacketLoss=0.01. Higher values of network’s QoE parameter are preferred.

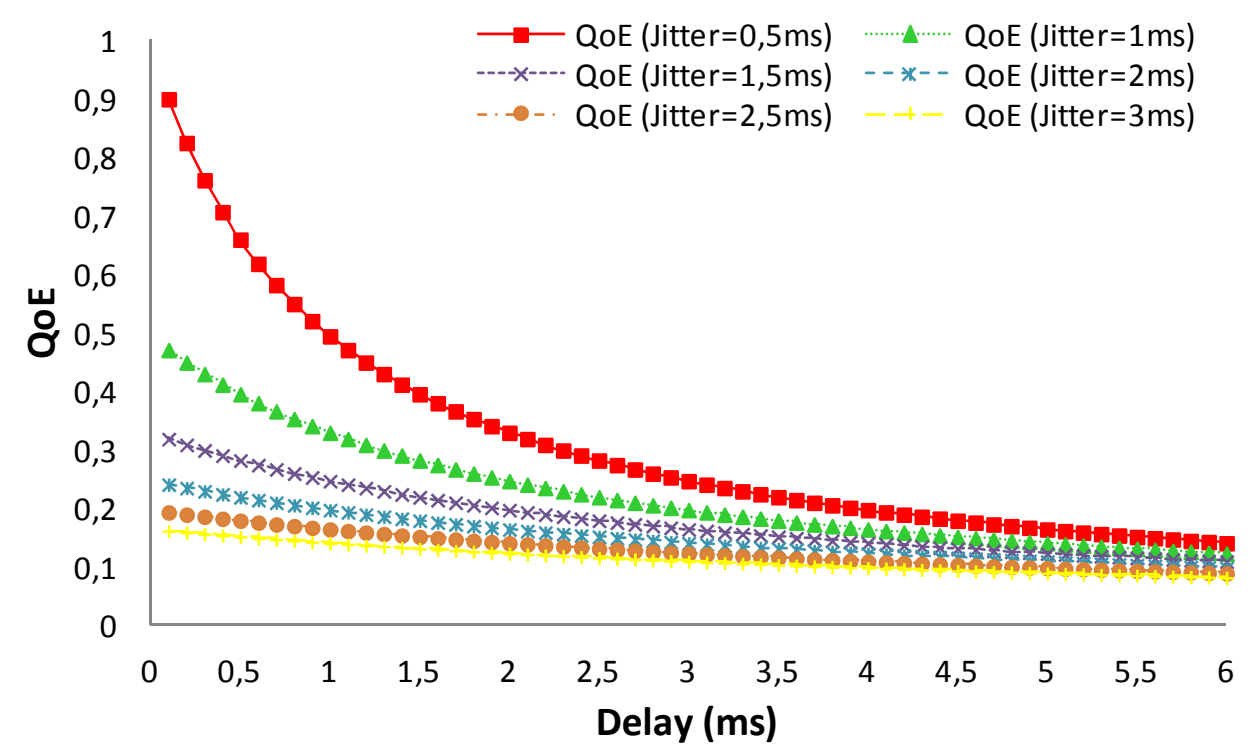

Figure 2. QoE values of the proposed formula.

When a user wants to watch 3D/2D IPTV, he/she opens the IPTV software which measures the wireless networks' RSSI in its coverage area and joins the one with highest value. Every time a device joins a wireless network it sends to the QoE test server the SSID and MAC address of the detected APs and the delay, jitter and lost packets taken from that network during 3 seconds. The QoE test server has a database with all wireless networks in the access network. Then, it sends a request to the QoE test server in order to test if there is enough available bandwidth to watch TV or Video on Demand. If there is not enough available bandwidth, the device adds this network to a discarded wireless networks list and joins the next one with highest RSSI value. If there is enough available bandwidth, it requests video streams to the IPTV server.

While the device is receiving the 3D/2D ITPV streams it also measures the delay, jitter and lost packets. This information is sent to the QoE test server which estimates the QoE for this user and compares it with the estimated QoE for other SSIDs of other APs. If the estimated QoE is higher than the others SSIDs under the coverage area of the client's device, the device remains in the same wireless network, but if it is lower, the QoE test server sends the SSID and the MAC address of the wireless network that has highest QoE to the device. Then, the device leaves its wireless network and joins the new one. Finally, the new customer sends a request to the IPTV server. 
Figure 3 shows the steps of the explained algorithm. The system described allows balancing the network’s QoE in by placing the customers to the best network’s QoE in that moment.

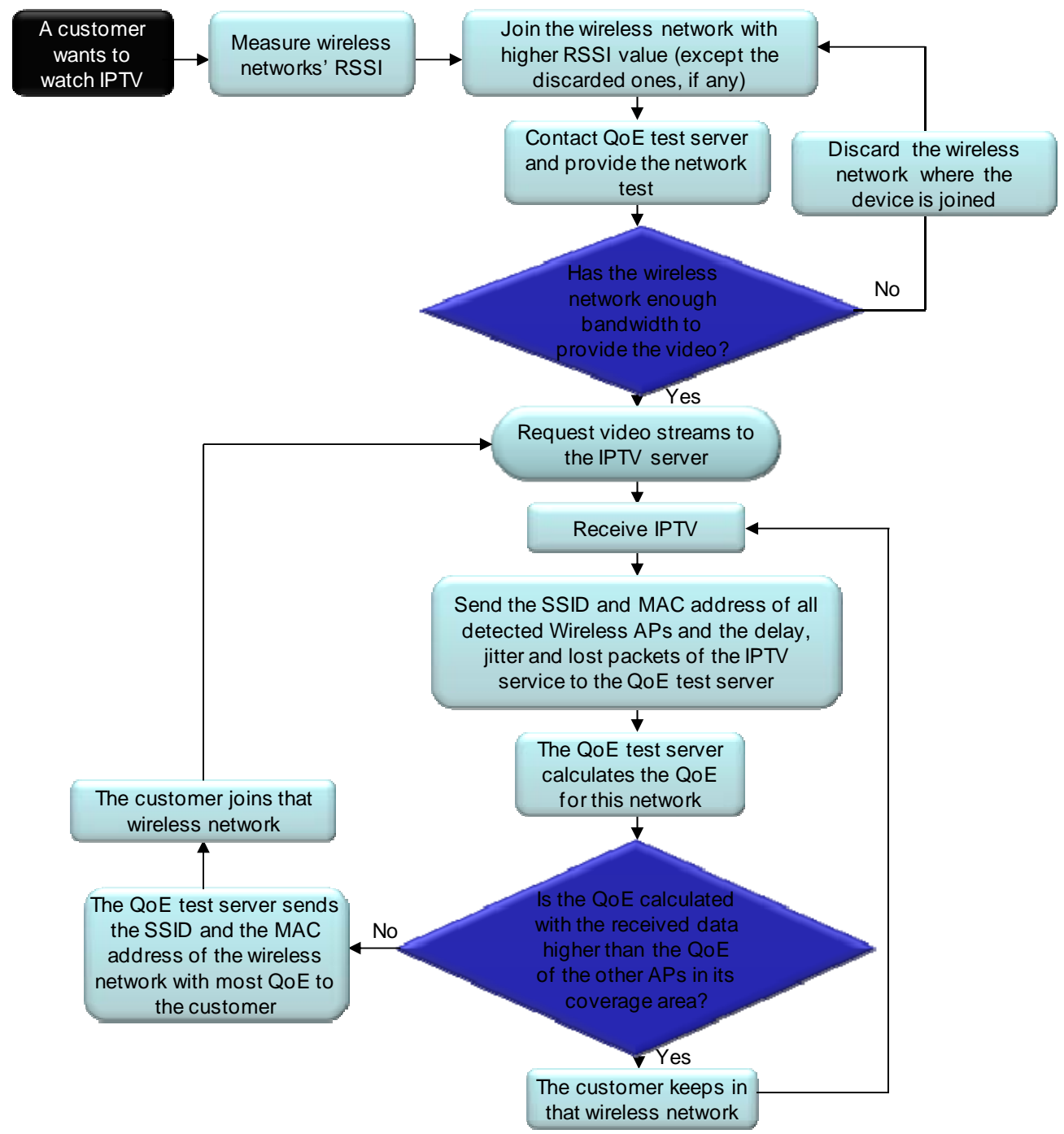

Figure 3. Proposed algorithm.

Figure 4 shows the protocol operation of the proposed algorithm. It shows the messages sent between the new customer and the QoE test server when the client's device joins a wireless network. When the QoE test server receives Join IPTV network message, it estimates the QoE of this network and compares it with the wireless networks under the coverage area of the customer. It also shows that if the QoE test server has a wireless network in its coverage area that has higher QoE values, it send a change wireless network message in order to let it know the appropriate wireless network to connect with. Finally, the client's device requests IPTV streams to the IPTV server though the request to the IPTV server message. 


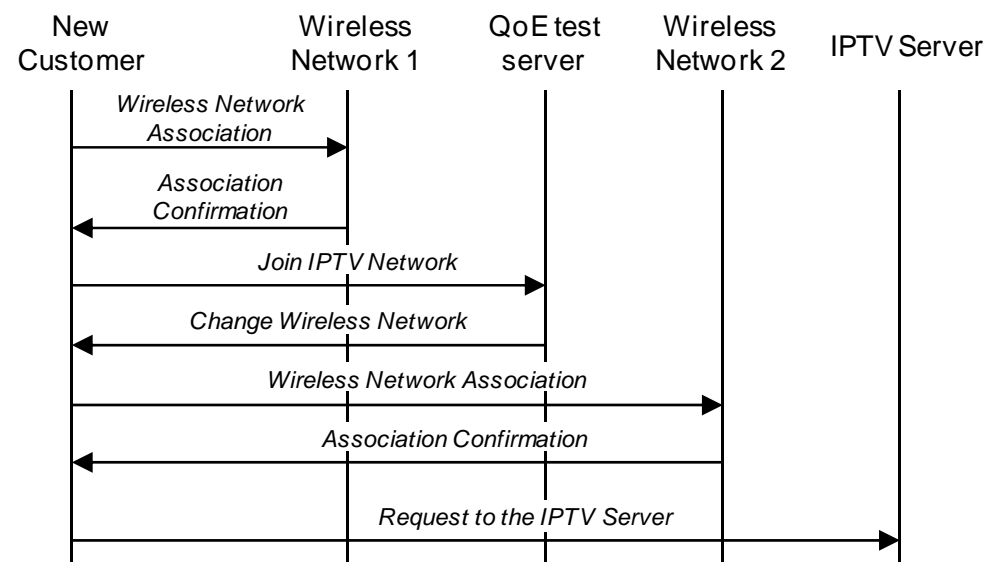

Figure 4. Messages sent in the proposed algorithm.

The system allows the customer to connect to the wireless network with better network's QoE. As new customers connect to any of the wireless networks, those with more users will experience higher delay, jitter and packet losses values, so network's QoE for that wireless networks will be lower and new users will choose one between the others. The system will balance the number of connected users to each network taking into account the network’s QoE for the user.

Although we will see in our performance test that the systems works successfully, when there are many clients, we can also include the algorithm proposed by us in [33] in order to predict the customer's mobility and reserve resources in the network devices to prevent the roaming and provide faster reconnections.

\section{Performance Test}

In this section we will show the measurements carried out in our experiment in order to evaluate the system performance.

\subsection{Test Bench}

In order to test the system performance and analyze which features offers, we used the scenario shown in figure 5. First, we used a multipoint to point WiMAX system. The WiMAX equipment is formed by an Alvarion BreezeACCESS VL Base Unit and an Alvarion BreezeACCESS VL Remote Bridge (working at the $5.4 \mathrm{GHz}$ frequency band). Second, we used a Lobometrics OSB Lobo 924N for IEEE WLAN 802.11a and an Intel Pro Wireless 3945 ABG wireless card in the laptop. Third, we used Linksys WAP54G Access-Point for IEEE WLAN 802.11g and an Intel Pro Wireless 3945 ABG wireless card in the laptop. Finally, forth, we used a Linksys WRT320N for the IEEE 802.11n and a Linksys WUSB600N wireless card in the laptop. 


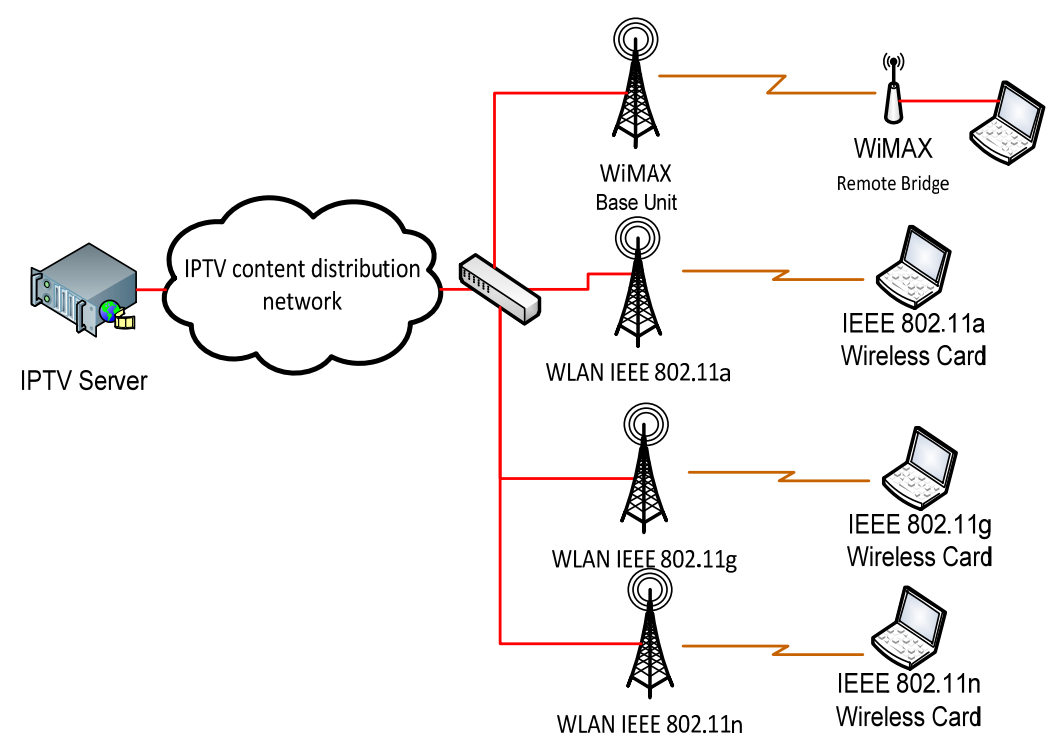

Figure 5. Network topology

We used the VLC Media Player [34], running in a Personal Computer, as the IPTV Server. The PC had a Fast Ethernet network interface card. It streamed HDTV 1080p (1920×1080 pixels) multicast video by using the following features: MPEG-TS video encapsulation, WMV (Windows Media Video) video format and (Windows Media Audio) WMA audio format. In order to perform our study, we captured RTP packets over UDP using the network protocol analyzer Wireshark [35].

\subsection{Video streaming test}

In order to analyze the performance and quality of our system, we streamed multicast HDTV 1080p video, and we tested the delay, jitter, bandwidth, and packet loss. The packets were captured by the laptop used as an IPTV client. This laptop was able to join to WiMAX, through the Alvarion BreezeACCESS VL Remote Bridge, and to the IEEE 802.11a/g/n by using Intel Pro Wireless 3945 ABG and Linksys WUSB600N wireless cards.

\subsubsection{Delay test}

Figure 6 shows the measured data for the delay test. In order to evaluate the performance, we analyze the packets received during a short period of time. We took 75 samples. IEEE 802.11g technology had the lowest average delay (1.28 msec.), while IEEE 802.11a had the highest (1.96 msec.), followed by WiMAX (1.94 msec.). Both the maximum peak (3.98 msec.) and the minimum peak (0.26 msec.) were given in IEEE 802.11n. It demonstrated that IEEE 802.11n had the worst behavior because its graph is the least uniform. We can see that the jitter was very similar in WiMAX and IEEE 802.11a. These technologies are approximately 35\% worst (in terms of delay) than IEEE 802.11g. 


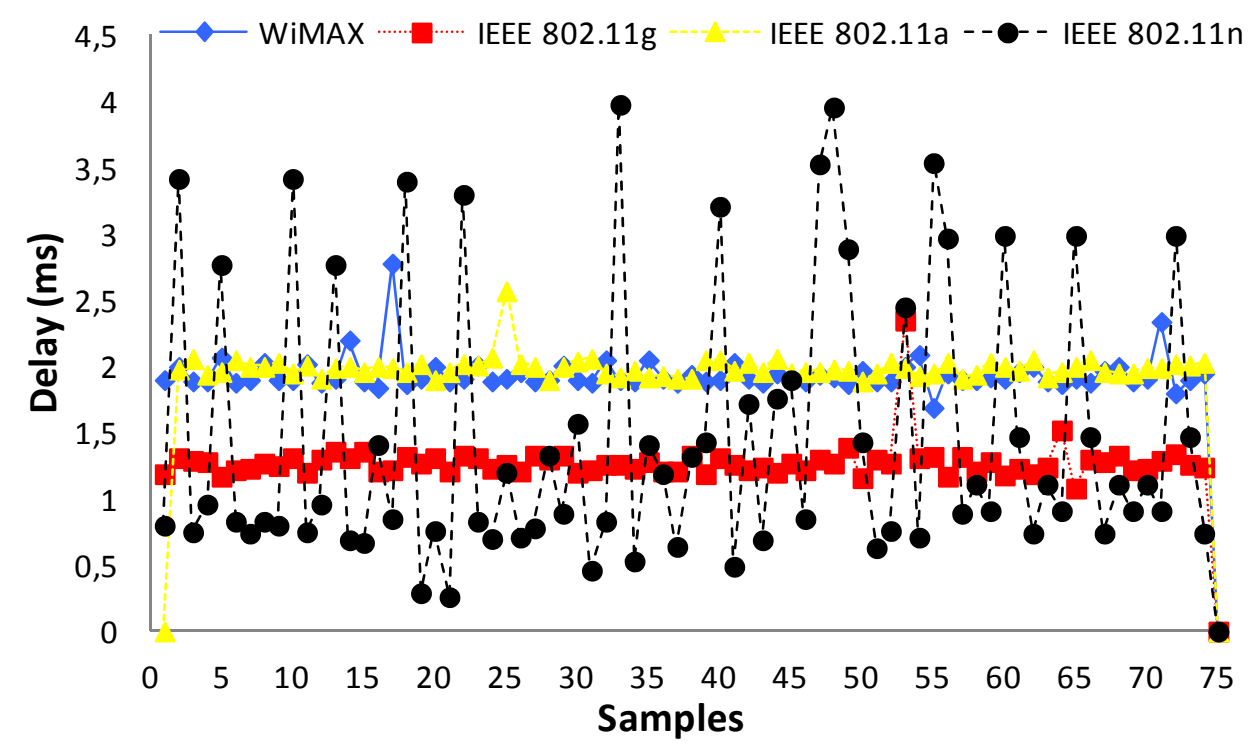

Figure 6. Delay test

We can see that all wireless technologies used give us an average delay in the range of 1.96 msec. and 1.28 msec.

\subsubsection{Jitter test}

Figure 7 shows the results of the jitter tests. The jitter is maintained almost constant all the time and lower than $0.5 \mathrm{msec}$. in all wireless technologies except IEEE 802.11n. The lowest jitter was obtained in WiMAX (0.30 msec.), followed by IEEE 802.11g ( 0.37 msec.). The highest jitter average has been obtained in IEEE 802.11n (3.61 msec.), which had its highest peak in $4.42 \mathrm{msec}$. The minimum jitter value was obtained in IEEE 802.11a (0.04 msec.), followed by WiMAX ( 0.27 msec.).

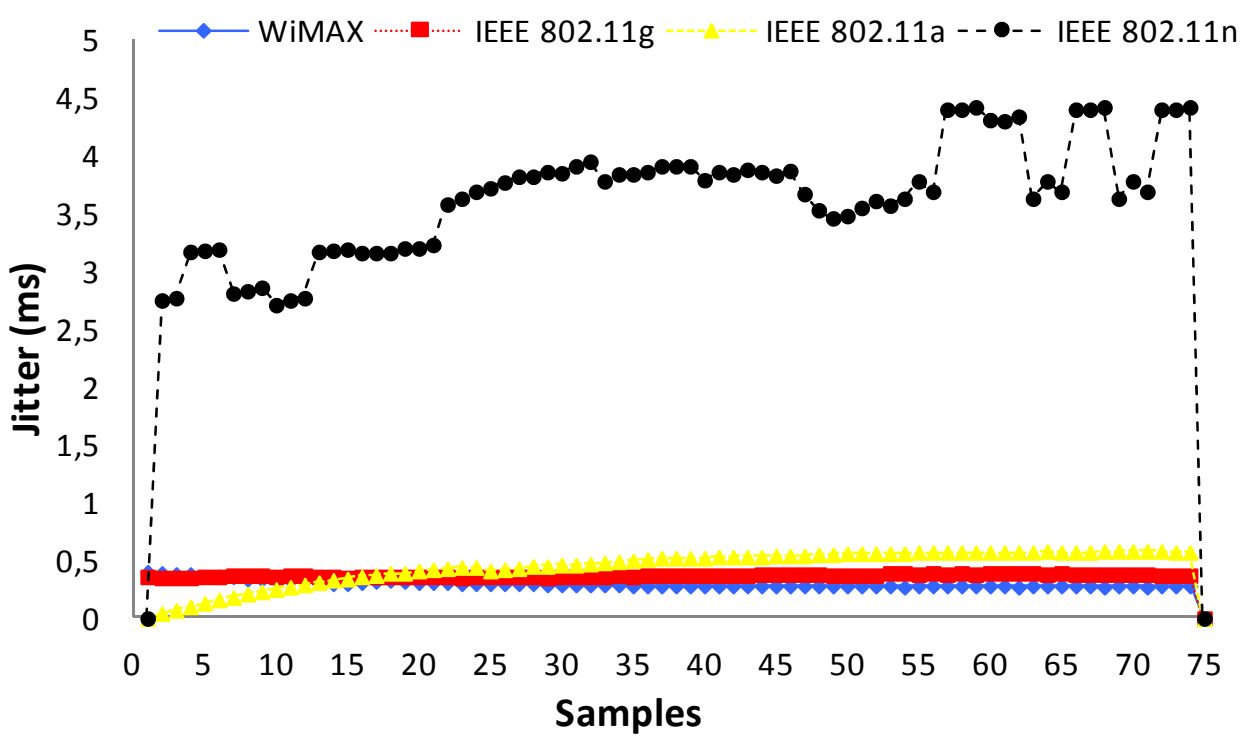

Figure 7. Jitter test 


\subsubsection{Lost packet test}

We observed that for 75 samples there was not any lost packet, so we performed a test that took 30 samples per second during 90 seconds. So, we took around 2700 samples. Figure 8 shows the measurements taken for this test. We can see that the wireless technologies with highest peaks are IEEE 802.11a and IEEE 802.11n.

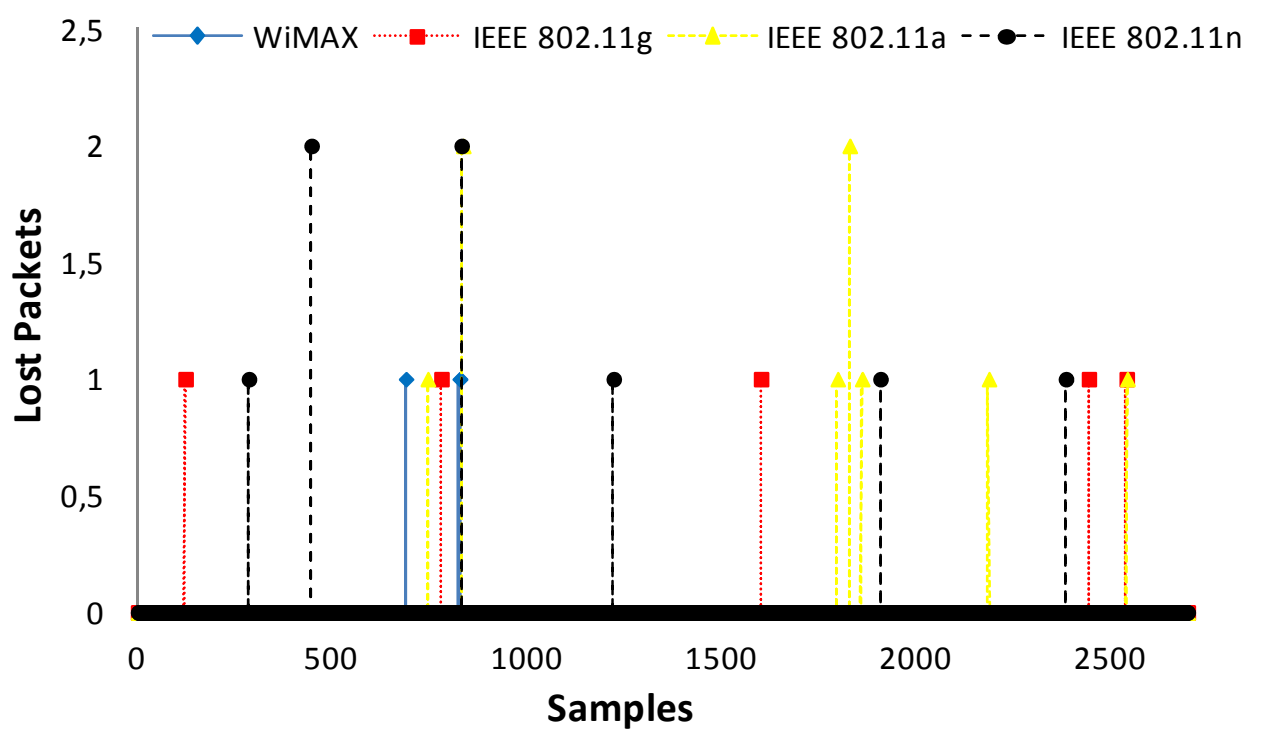

Figure 8. Lost Packets test

Then, we analyzed the number of total lost packets. Table 4 shows the summary. The WiMAX technology had an average value of 3 packets ( $0.02 \%$ of lost packets). On the other hand, IEEE 802.11g had an average of 5 lost packets ( $0.04 \%$ of lost packets). Finally, the worst case was IEEE 802.11a. In this case we obtained 9 lost packets (0.33\%). It seems that the IEEE 802.11a and IEEE 802.11n technologies are less robust than the other ones.

Table 4. Lost Packets.

\begin{tabular}{lcccc} 
& WiMAX & IEEE 802.11g & IEEE 802.11a & IEEE 802.11n \\
\hline Lost Packet & 3 & 5 & 9 & 8 \\
Lost Packet (\%) & 0,02 & 0,04 & 0,33 & 0,30
\end{tabular}

\subsection{Effective bandwidth test}

Figure 9 shows the test of the effective bandwidth for all technologies in a real environment. We have compared it with the bandwidth consumed by the streamed video (Video BW). It has an average value of $849.90 \mathrm{Kbps}$. These tests show that, empirically, a mean maximum of 4744.21 Kbps can be transmitted in WiMAX, a mean maximum of 2714.16 Kbps in IEEE 802.11n, a mean maximum of 2314.69 Kbps in the IEEE 802.11g and a mean maximum of $1183.40 \mathrm{Kbps}$ in IEEE 802.11a. This is very important when we estimate the theoretical number IPTV channels that can be streamed by the IPTV server. Thus, the theoretical number of IPTV channels with a HDTV 
1080p quality of video that our IPTV server can support is 5 IPTV channels in WiMAX, 3 IPTV channels in IEEE 802.11n, 2 IPTV channels in the IEEE 802.11g, and only one channel for IEEE 802.11a.

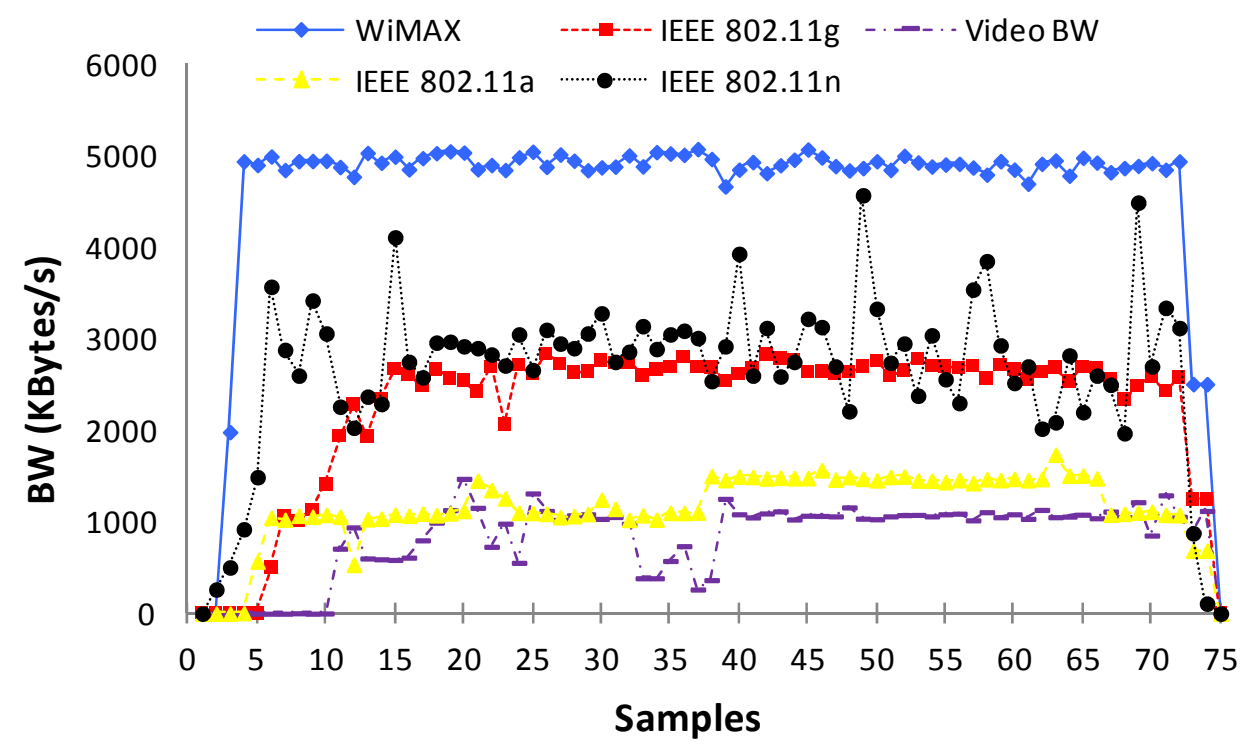

Figure 9. Effective bandwidth and video bandwidth test

\subsection{QoE comparison}

In figure 10, the QoE parameter for the four wireless networks is shown. IEEE 802.11a technology has a very stable network's QoE (because it has less number of peaks). The best QoE average is obtained for IEEE 802.11g (0.497). This technology also had the maximum value (0.538). The worst case is obtained for IEEE 802.11n, which had an average value of 0.116 (which a minimum value of 0.074). WiMAX and IEEE 802.11a had close values. We have also observed during our test that when there are more clients in the network, QoE values decrease because there are worse conditions of jitter, delay and lost packets.

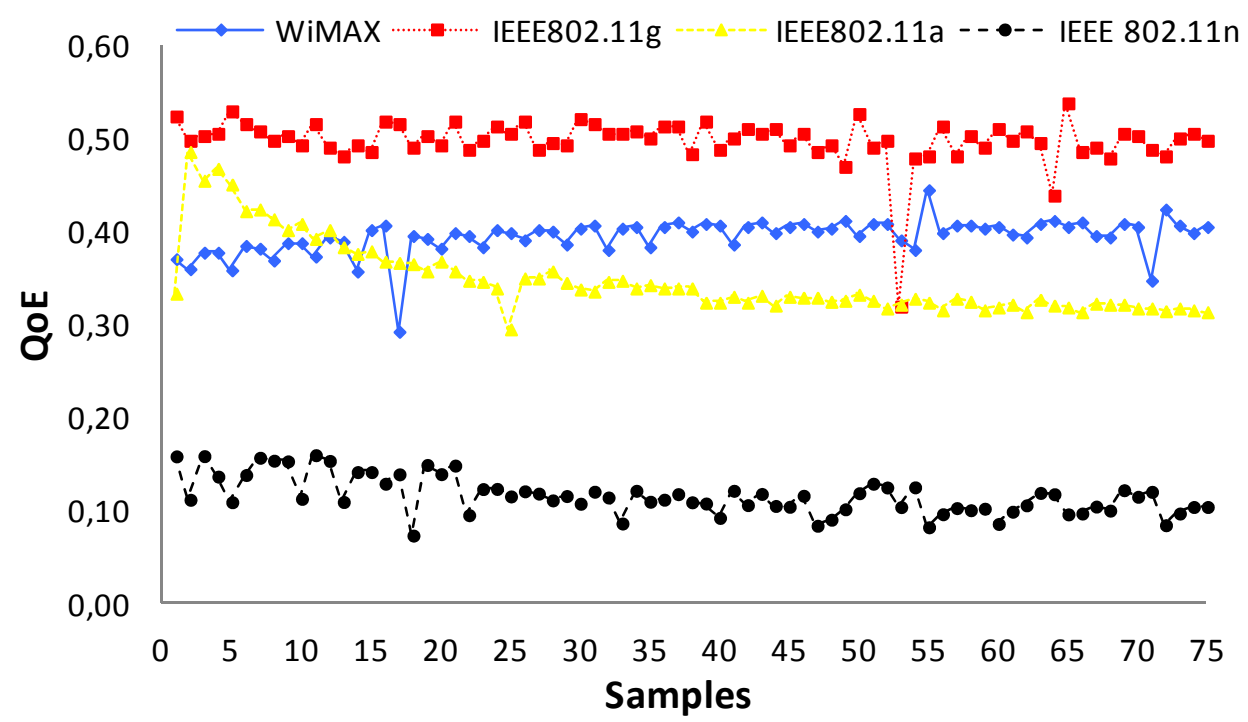

Figure 10. QoE test 


\subsection{Roaming test}

In order to test the performance of the system when the customer roams from one wireless technology to another, we have measured the packet loss, the jitter, the delay, and the number of frames received by the client device every second. The video used for our test bench is described in subsection 5.1. In order to avoid any dependence with the type of device, the trademark or the model, we have used only one type of access point that allows us to roam between several wireless technologies. In this case, we used Linksys WRT320N, which allowed us to roam between IEEE 802.11a/g/n and a Linksys WUSB600N wireless card for the client device. In order to analyze the behavior of the roaming and its impact on the end user in detail, we show the graphs of the number of lost packets, delay and jitter placing the exact moment of the roaming (that is when there are more packets lost) approximately in the $50^{\text {th }}$ sample and we show 50 samples before and 50 samples after that moment. Finally, we will show the number of packets transmitted in each second when the roaming is taking place (during 20 seconds).

Figure 11 shows the number of parquets lost during the 100 samples. The worst case has been in the roaming between IEEE 802.11g and IEEE 802.11a (with 18 packets lost), followed by the roaming between IEEE 802.11a and IEEE 802.11g (with 17 packets lost). It seems to be because there is a frequency change between $2.4 \mathrm{GHz}$ and $5 \mathrm{GHz}$ and vice versa.

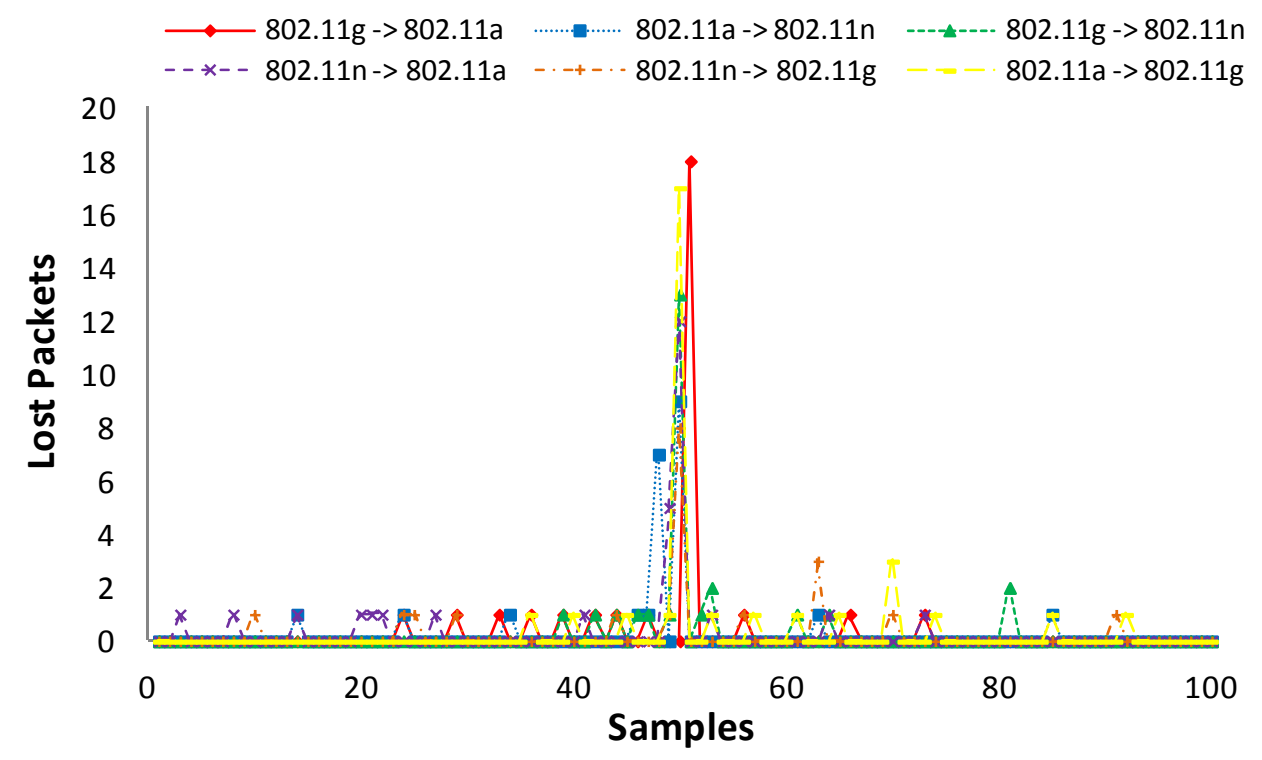

Figure 11. Lost Packets test comparison

When we compare the delay when the roaming is being performed (see figure 12), the worst cases seem to be when IEEE 802.11a is involved in the roaming. The highest delay was 19.88 msec. However, the roaming between IEEE 802.11g and IEEE 802.11a did not provided any strange peak. The best delay results have been obtained for the roaming between IEEE 802.11n and IEEE 802.11g and vice versa. 


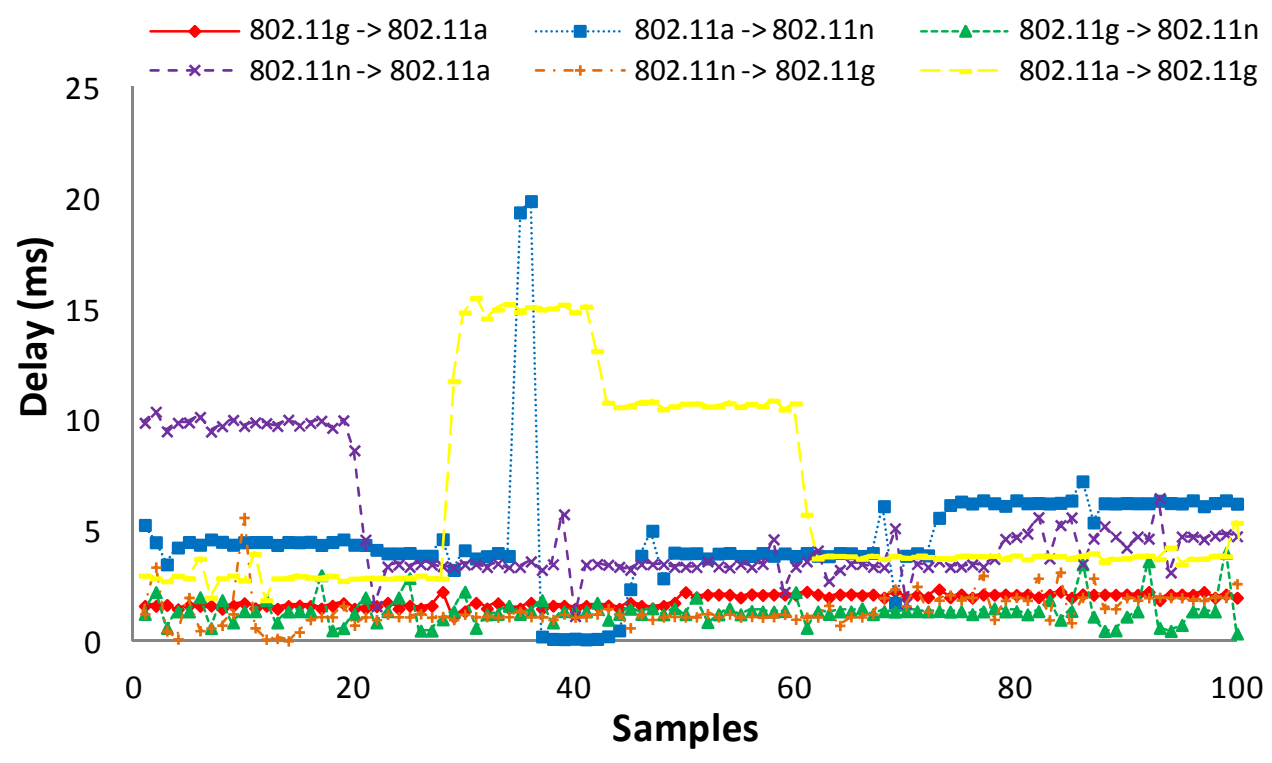

Figure 12. Delay test comparison

In the Jitter test (see figure 13), except the roaming between 802.11a and 802.11n, which reached a peak of 2.73 msec., the jitter has been maintained below $0.7 \mathrm{msec}$. all the time for the rest of the cases. It allows us to know that the customer will not appreciate the impact of the roaming when he/she is watching IPTV. Moreover, for extreme cases, we can increase the buffer of the IPTV player in order to decrease any type of impact in the end user QoE.

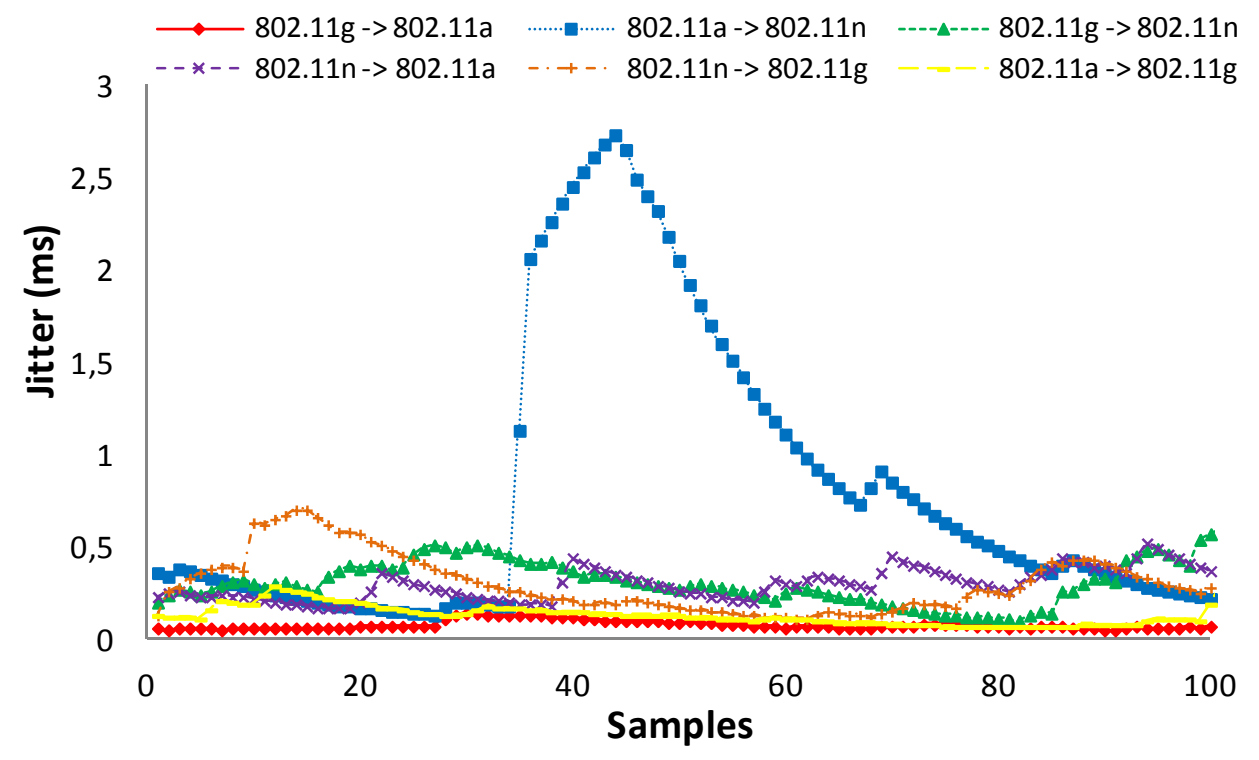

Figure 13. Jitter test comparison

Figure 14 shows the number of IPTV packets receiver at the customer's device when the roaming is being performed in the $10^{\text {th }}$ second. We can see that although a reduction of the number of packets can be appreciated between the $10^{\text {th }}$ and the $14^{\text {th }}$ second in almost all cases, this behaviour does not have any impact in the end user view. 


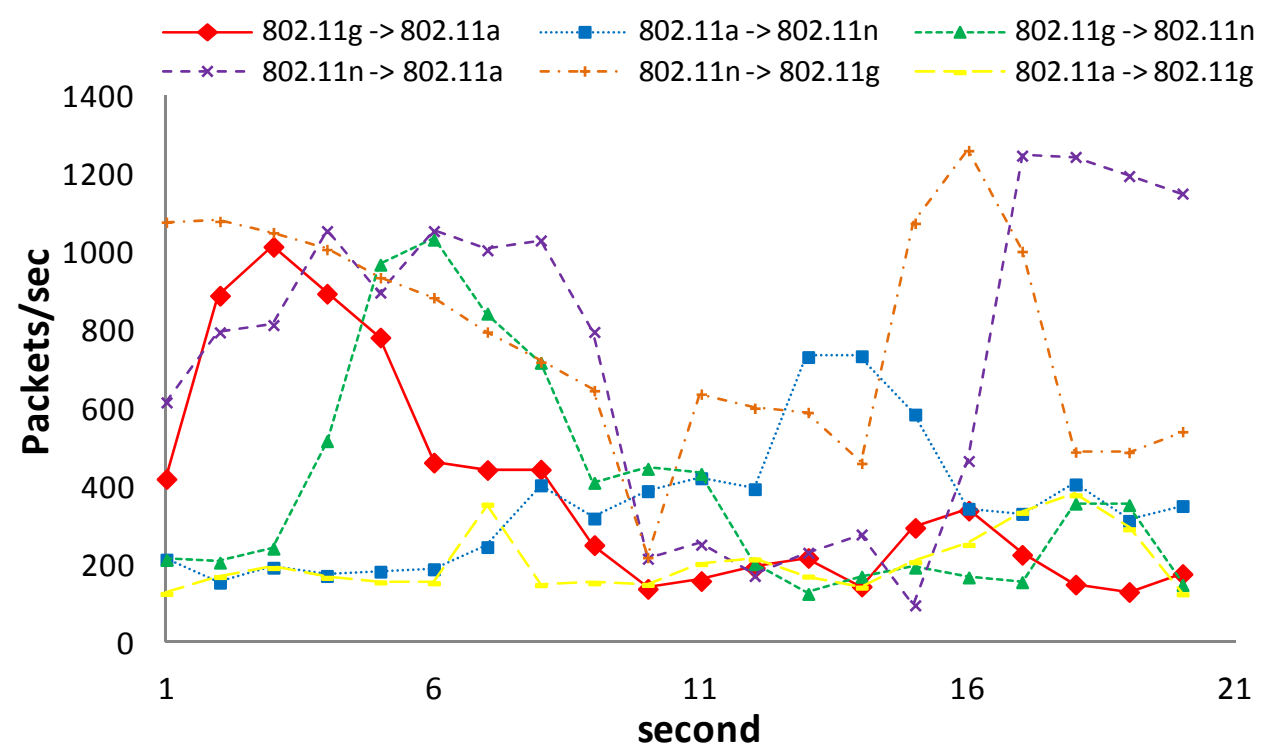

Figure 14. Packets per second test comparison

\section{Conclusion}

In this paper we propose a new network algorithm for IPTV distribution using WiMAX and WLAN technologies. It chooses the appropriate wireless access technology in order to obtain higher QoE parameter for IPTV. It ensures to have the best QoE at the customer's side. We have described the algorithm and explained the proposed protocol for its proper operation. Obtained measurements show the comparison of delay, jitter and lost packets received by the client's device when the system selects each one of the technologies included in our system. We have also shown the effective bandwidth and the bandwidth consumed by one IPTV HDTV channel. In terms of bandwidth, the most appropriate is WiMAX, but IEEE 802.11g provides better QoE results. The worst technology has been IEEE 802.11n. It may happen because the device used to perform the measurements had implemented IEEE 802.11n Draft. When we tested the impact of the user when the device is roaming between technologies, we observed that the worst case in the delay is when IEEE 802.11a technology is involved in the roaming, but in no case the end user had an appreciation while watching TV. Now we are improving our IPTV client in order to achieve lower zapping time delays (that is when the client changes the multicast group, the channel). Moreover we are working on including more parameters such as round trip delay, video Quality and audio quality, in order to fine the algorithm. Future studies will include other wireless technologies such as GSM, LTE, etc. Our proposal can also be used in any IP multimedia subsystem [36] in order to achieve better QoE for the multimedia devices.

\section{Acknowledgments}

This work has been partially supported by the Polytechnic University of Valencia, though the PAID-15-10 multidisciplinary projects, by the Instituto de Telecomunicações, Next Generation Networks and Applications Group (NetGNA), Portugal, and by National Funding from the FCT Fundação para a Ciência e a Tecnologia through the PEst-OE/EEI/LA0008/2011 Project. 


\section{References}

[1] C. Hellberg, D. Greene, T. Boyes, "Broadband network architectures: designing and deploying tripleplay services”, Prentice Hall PTR Upper Saddle River, NJ, USA, 2007.

[2] inCode Telecom group Inc. The Quad-Play - the First Wave of the Converged Services Evolution. White paper, February 2006.

[3] K. Knightson, N. Morita, T. Towle, "NGN architecture: generic principles, functional architecture, and implementation,” IEEE Communications Magazine, vol.43, no.10, pp. 49-56, Oct. 2005.

[4] G. Schollmeier, C. Winkler, "Providing sustainable QoS in next-generation networks,” IEEE Communication Magazine, vol.42, nº 6, pp. 102-107, June 2004.

[5] B. Alfonsi, “I want my IPTV: Internet Protocol television predicted a winner,” IEEE Distributed Systems Online, vol.6, no.2, Feb. 2005

[6] IPTV Focus Group, Available at http://www.itu.int/ITU-T/IPTV/ [last access: July 2011]

[7] Y. Xiao, X. Du, J. Zhang, Fei Hu, S. Guizani, Internet protocol television (IPTV): the killer application for the next-generation internet, IEEE Communications Magazine. Vol. 45, Issue 11. Pp. 126-134. November 2007.

[8] F. E. Retnasothie, M. K. Ozdemir, T. YÄucek, J. Zhang, H. Celebi, and R. Muththaiah, "Wireless IPTV over WiMAX: Challenges and applications”. IEEE Wamicon, Clearwater, FL, Dec. 2006.

[9] J. She, Fen Hou, Pin-Han Ho, Liang-Liang Xie, "IPTV over WiMAX: Key Success Factors, Challenges, and Solutions [Advances in Mobile Multimedia],” IEEE Communications Magazine, vol.45, no.8, pp.87-93, August 2007.

[10] Abdulrahman Yarali, Saifur Rahman, Bwanga Mbula, WIMAX: The innovate Broadband Wireless access technology, Journal of Communications, Vol 3, No 2 (2008), 53-63, April 2008.

[11] Wen-Hsing Kuo, Tehuang Liu, Wanjiun Liao, Utility-Based Resource Allocation for Layer-Encoded IPTV Multicast in IEEE 802.16 (WiMAX) Wireless Networks. IEEE International Conference on Communications 2007 (ICC 2007), 24-28 June 2007. Glasgow, Scotland. Pp.1754-1759

[12] H. Singh, ChangYeul Kvvon; Seong Soo Kim; Chiu Ngo, "IPTV over WirelessLAN: Promises and Challenges,” 5th IEEE Consumer Communications and Networking Conference, Pp.626-631, January 2008

[13] E. Shihab, F. Wan, L. Cai, A. Gulliver and N. Tin "Performance Analysis of IPTV in Home Networks", IEEE Global Telecommunications (GLOBECOM 2007), Washington, DC, 26-30 November 2007.

[14] E. Shihab, L. Cai, F. Wan, T. A. Gulliver, and N. Tin, "Wireless mesh networks for in-home IPTV distribution,” IEEE Network, vol. 22, no. 1, pp. 52-57, Jan.-Feb. 2008.

[15] M. Gidlund, J. Ekling, "VoIP and IPTV distribution over wireless mesh networks in indoor environment,” IEEE Transactions on Consumer Electronics, vol.54, no.4, pp.1665-1671, November 2008

[16] Jin-Yu Zhang; Man-Gui Liang, "IPTV QoS Implement Mechanism in WLAN," Int. Conference on Intelligent Information Hiding and Multimedia Signal Processing. pp.117-120, 15-17 August 2008

[17] A. H. Park and J. K. Choi, “QoS guaranteed IPTV service over Wireless Broadband network”, The 9th Int. Conference on Advanced Communication Technology, vol. 2, pp. 1077--1080, February 2007.

[18] K.-H. Lee; S. T. Trong, B.-G. Lee; Y.-T. Kim, QoS-Guaranteed IPTV Service Provisioning in Home Network with IEEE 802.11e Wireless LAN,” IEEE Network Operations and Management Symposium. Pp.71-76, April 2008.

[19] F. Birlik, Ö. Gurbuz and O. Ercetin. IPTV Home Networking via 802.11 Wireless Mesh Networks: An Implementation Experience. IEEE Trans. on Consumer Electronics, Vol. 55, No. 3, August 2009.

[20] G.Cunningham, P.Perry, J.Murphy, L.Murphy. Seamless Handover of IPTV Streams in a Wireless LAN Network. Transactions on Broadcasting, IEEE. Vol. 55, Issue 4. Pp. 796 - 801, Dec. 2009.

[21] S. Abukharis, R. MacKenzie and T. O Farrell, 2009. Improving QoS of Video Transmitted Over 802.11 WLANs Using Frame Aggregation. London Communications Symposium.. London, United Kingdom, September 03 - 04, 2009.

[22] L. X. Cai, X. Ling, X. Shen, J. W. Mark and L. Cai, Supporting voice and video applications over IEEE 802.11n WLANs. Wireless Networks, Vol 15, pp 443-454. 2009.

[23] Marcelo Atenas, Sandra Sendra, Miguel Garcia, Jaime Lloret, IPTV Performance in IEEE 802.11n WLANs, IEEE Global Communications Conference (IEEE Globecomm 2010), Miami (USA), December 6-10, 2010.

[24] Heng-Tung Hsu, Fang-Yao Kuo, Ping-Hung Lu, Design of WiFi/WiMAX dual-band E-shaped patch antennas through cavity model approach, Microwave and Optical Technology Letters, Volume 52, Issue 2, pages 471-474. February 2010. 
[25] S. Jindal, A. Jindal, N. Gupta, Grouping WI-MAX, 3G and WI-FI for wireless broadband, The First IEEE and IFIP International Conference in Central Asia on Internet 2005, September 26-29, 2005, Bishkek, Kyrgyzstan.

[26] Z. Dai, R. Fracchia, J. Gosteau, P. Pellati, G. Vivier, Vertical Handover Criteria and Algorithm in IEEE802.11 and 802.16 Hybrid Networks, IEEE International Conference on Communications, 2008. ICC '08. Beijing, China, 19-23 May 2008.

[27] Miguel Garcia, Jaime Lloret, Miguel Edo, Raquel Lacuesta, IPTV Distribution Network Access System Using WiMAX and WLAN Technologies, International Symposium on High Performance Distributed Computing (HPDC 2009), Munich (Germany), June 11-13, 2009.

[28] Karen Fernanda Medina Velez and Ivonne Alexandra Revelo Arias, Diseño y planificación de una red inalámbrica basada en los estandares IEEE 802.16 (WIMAX) y 802.11 (WIFI) para proveer de internet de banda ancha a poblaciones de las provincias de Loja y Zamora Chinchipe, Tesis Electrónica y Telecomunicaciones (IET), Escuela Politécnica Nacional, Quito, Ecuador, August 2006.

[29] IEEE Std 802.16 ${ }^{\mathrm{TM}}$-2009, IEEE Standard for Local and metropolitan area networks, Part 16: Air Interface for Broadband Wireless Access Systems. At http://standards.ieee.org/getieee802/download/802.16-2009.pdf [last access: July 2011]

[30] IEEE 802.11 Working Group, At http://www.ieee802.org/11/index.shtml [last access: July 2011]

[31] IEEE Std 802.11 information exchange between systems - Local and metropolitan area networks- Specific requirements Part 11: Wireless LAN Medium Access Control (MAC) and Physical Layer (PHY) Specifications.

[32] Super AG technologies, At http://www.digicom.it/italiano/supporto/WhitePaper/Wireless108M_whitepaper.pdf [last access: July 2011]

[33] Alejandro Canovas, Fernando Boronat, Carlos Turro and Jaime Lloret, Multicast TV over WLAN in a University Campus Network, The Fifth International Conference on Networking and Services (ICNS 2009), Valencia (Spain), April 20-25, 2009.

[34] VLC Media Player, Available at www.videolan.org [last access: July 2011]

[35] Wireshark Network Protocol Analyzer, Available at www.wireshark.org [last access: July 2011]

[36] C. Lai, Min Chen, Playback-Rate Based Streaming Services for Maximum Network Capacity in IP Multimedia Subsystem, IEEE System Journal, DOI : 10.1109/JSYST.2011.2165190, 2011. 